POS PROCEEDINGS

\title{
Nuclear effective field theory on the lattice
}

\section{Hermann Krebs ${ }^{* a, c}$}

E-mail: h.krebs@fz-juelich.de

\section{Buḡra Borasoy ${ }^{a}$}

E-mail: borasoyeitkp.uni-bonn. de

\section{Evgeny Epelbaum ${ }^{c, a}$}

E-mail: e.epelbaumefz-juelich.de

\section{Dean Lee ${ }^{d}$}

E-mail: djlee3@ncsu.edu

Ulf-G. Meißner ${ }^{a, b, c}$

E-mail: meissnerditkp.uni-bonn.de

${ }^{a}$ Helmholtz-Institut für Strahlen- und Kernphysik (Theorie), Universität Bonn, Nußallee 14-16, D-53115 Bonn, Germany

${ }^{b}$ Bethe Center for Theoretical Physics, Universität Bonn, D-53115 Bonn, Germany

${ }^{c}$ Institut für Kernphysik (IKP-3) and Jülich Center for Hadron Physics, D-52425 Jülich, Germany

${ }^{d}$ Department of Physics, North Carolina State University, Raleigh, NC 27695, USA

In the low-energy region far below the chiral symmetry breaking scale of $\Lambda_{\chi} \sim 1 \mathrm{GeV}$ chiral perturbation theory (ChPT) provides a model-independent approach for quantitative description of nuclear processes. In the two- and more-nucleon sector perturbation theory is applicable only at the level of an effective potential which serves as input in the corresponding dynamical equation. To deal with the resulting many-body problem we put chiral effective field theory (EFT) on the lattice. Here we present the results of our lattice EFT study up to next-to-next-to-leading order $\left(\mathrm{N}^{2} \mathrm{LO}\right)$ in the chiral expansion. Accurate description of two-nucleon phase-shifts and ground state energy ratio of dilute neutron matter up to corrections of higher orders shows that lattice EFT is a promising tool for a quantitative description of low-energy few- and many-body systems.

The XXVI International Symposium on Lattice Field Theory

July 14-19 2008

Williamsburg, Virginia, USA

\footnotetext{
*Speaker.
} 


\section{Introduction}

Quantum chromodynamics (QCD) describes the interaction between quarks and gluons which is responsible for the strong nuclear force. Recent advances in QCD using computational lattice methods have made it possible to accurately predict the spectrum and properties of many isolated hadrons. Unfortunately, lattice QCD calculations of nuclear and neutron matter or even few-body systems beyond two nucleons are presently not possible. The most significant challenge in such simulations would be to overcome the exponentially small signal-to-noise ratio caused by the sign and complex phase oscillations for simulations at large quark number.

Nuclear lattice simulations based on EFT provide an alternative method to describe few- and many-body systems at low energy without losing connection to QCD. The lattice EFT approach addresses the few- and many-body problem in nuclear physics by applying non-perturbative lattice methods to low-energy nucleons and pions. The effective Lagrangian is formulated on a spacetime lattice and the path integral is evaluated by Monte Carlo sampling. Pions and nucleons are treated as point-like particles on the lattice sites. By using hadronic degrees of freedom and concentrating on low-energy physics, it is possible to probe large volumes and greater number of nucleons than in lattice QCD. After a brief overview what has been done in this field so far we present some results of our recent studies of the two nucleon system [1][2] and neutron matter [3] at subleading order. Accurate description of two-nucleon phase-shifts and ground state energy ratio of dilute neutron matter up to corrections of higher orders show that lattice EFT is a promising tool for quantitative studies of low-energy few- and many-body systems.

\section{Lattice EFT: previous achievements}

Lattice EFT is a rather new and fast developing field. Here we give a brief overview on what has been done in this field so far. For a comprehensive discussion the reader is referred to [4]. The first lattice study of nuclear matter was carried out in the early nineties by Brockman and Frank [5] using a momentum lattice and based on the hadrodynamics model of Walecka [6]. The first nuclear lattice calculation based on EFT was carried out by Müller et al. [7]. They looked at infinite nuclear and neutron matter at nonzero density and temperature. Later a series of analytical studies were carried out: Chen and Kaplan [8] showed the absence of sign oscillation for nonzero chemical potential in the Hubbard model. Non-linear realization of chiral symmetry with static nucleons on the lattice was discussed by Chandrasekharan et al. [9]. Also ChPT within the lattice regularization was considered by several groups $[10,11,12]$. This was followed by the first many-body lattice calculation using chiral EFT [13]. Since that time a number of lattice calculations for cold atoms and low-energy nuclear physics were carried out. See [4] for a review article. It is important to note that in the low-energy sector the phase region accessible by lattice EFT is much broader than in lattice QCD. Severe sign oscillation problem limits the accessibility of finite density lattice QCD simulations. In contrast, sign oscillations in nuclear lattice EFT are strongly suppressed due to the approximate SU(4)-symmetry in the two-nucleon sector. One can show explicitely that SU(4)symmetric nuclear EFT does not have a sign problem. SU(4) symmetry breaking leads to small sign oscillations which, however, turns out to be not severe. 


\begin{tabular}{||l||c|c|c||c||}
\hline \hline & NLO & $\mathrm{N}^{2} \mathrm{LO}$ & $\mathrm{N}^{3} \mathrm{LO}$ & $\operatorname{Exp}$ \\
\hline \hline$E_{\mathrm{d}}[\mathrm{MeV}]$ & $-2.171 \ldots-2.186$ & $-2.189 \ldots-2.202$ & $-2.216 \ldots-2.223$ & $-2.224575(9)$ \\
$\eta_{\mathrm{d}}$ & $0.0256 \ldots 0.0257$ & $0.0255 \ldots 0.0256$ & $0.0254 \ldots 0.0255$ & $0.0256(4)$ \\
$A_{S}\left[\mathrm{fm}^{-1 / 2}\right]$ & $0.868 \ldots 0.873$ & $0.874 \ldots 0.879$ & $0.882 \ldots 0.883$ & $0.8846(9)$ \\
\hline \hline
\end{tabular}

Table 1: Deuteron properties at NLO, $\mathrm{N}^{2} \mathrm{LO}$ and $\mathrm{N}^{3} \mathrm{LO}$ compared to the data. Here, $E_{\mathrm{d}}$ is the binding energy, $\eta_{\mathrm{d}}$ the asymptotic $D / S$ ratio and $A_{S}$ the strength of the asymptotic S-wave normalization. The data for $E_{\mathrm{d}}$ are from [23], for $\eta_{\mathrm{d}}$ from [24] and for $A_{S}$ from [25].

\section{Nuclear EFT}

Let us now give a brief introduction to the basic foundations of our approach. The low-energy properties of hadronic systems are, in principle, accessible in lattice QCD. This method is, however, very expensive, especially if one wants to consider few- and many-nucleon systems. Alternatively, we can exploit the spontaneously broken approximate chiral symmetry of QCD which implies the existence of light weakly interacting Goldstone bosons. In the SU(2) sector, we identify the Goldstone bosons with pions. Since the interaction between the Goldstone bosons is weak one can apply perturbation theory, where the expansion parameter is not a coupling constant but small momenta and masses of the Goldstone bosons divided by the chiral symmetry breaking scale $\Lambda_{\chi}$. This systematic procedure is called chiral perturbation theory [14] and reproduces (as explicitely proved by Leutwyler [15] in mesonic sector) order by order the original QCD Green-functions.

ChPT has been extended to one nucleon sector. In the two and more nucleon sector additional problems appear. Due to the existence of nuclear bound states, the strict perturbative procedure breaks down. As shown by Weinberg [16], the power counting is violated by nucleon-nucleon (NN) cuts. He suggested to construct perturbatively a so-called chiral effective potential which, per construction, excludes the NN cuts and, for this reason does not violate the power counting of ChPT. To describe nuclear observables in the two-, three- or more-nucleon sectors one should numerically solve the Lippmann-Schwinger, Faddeev or Faddeev-Jakubowsky equations, respectively, with the chiral effective potential as an input.

Chiral effective potential has been extensively studied in the last decade up to next-to-next-tonext-to-leading order $\left(\mathrm{N}^{3} \mathrm{LO}\right)$ in chiral expansion (for extensive discussion see [17]). At this order two leading-order (LO), seven subleading order (NLO) and fifteen $\mathrm{N}^{3} \mathrm{LO}$ unknown low energy constants have been fitted to low energy nucleon data $[18,19,20]$. At this order in the chiral expansion, one observes an accurate description of all NN low-energy observables, see Figs. 1, 2 and Table 1.

\section{Nuclear EFT on the lattice}

Once the chiral nuclear forces are determined and the low energy constants appearing in the nuclear forces are fitted (in the two and three-nucleon sector) one can make predictions in the four- and more-nucleon sectors based on chiral EFT. However, explicit numerical treatment of the 

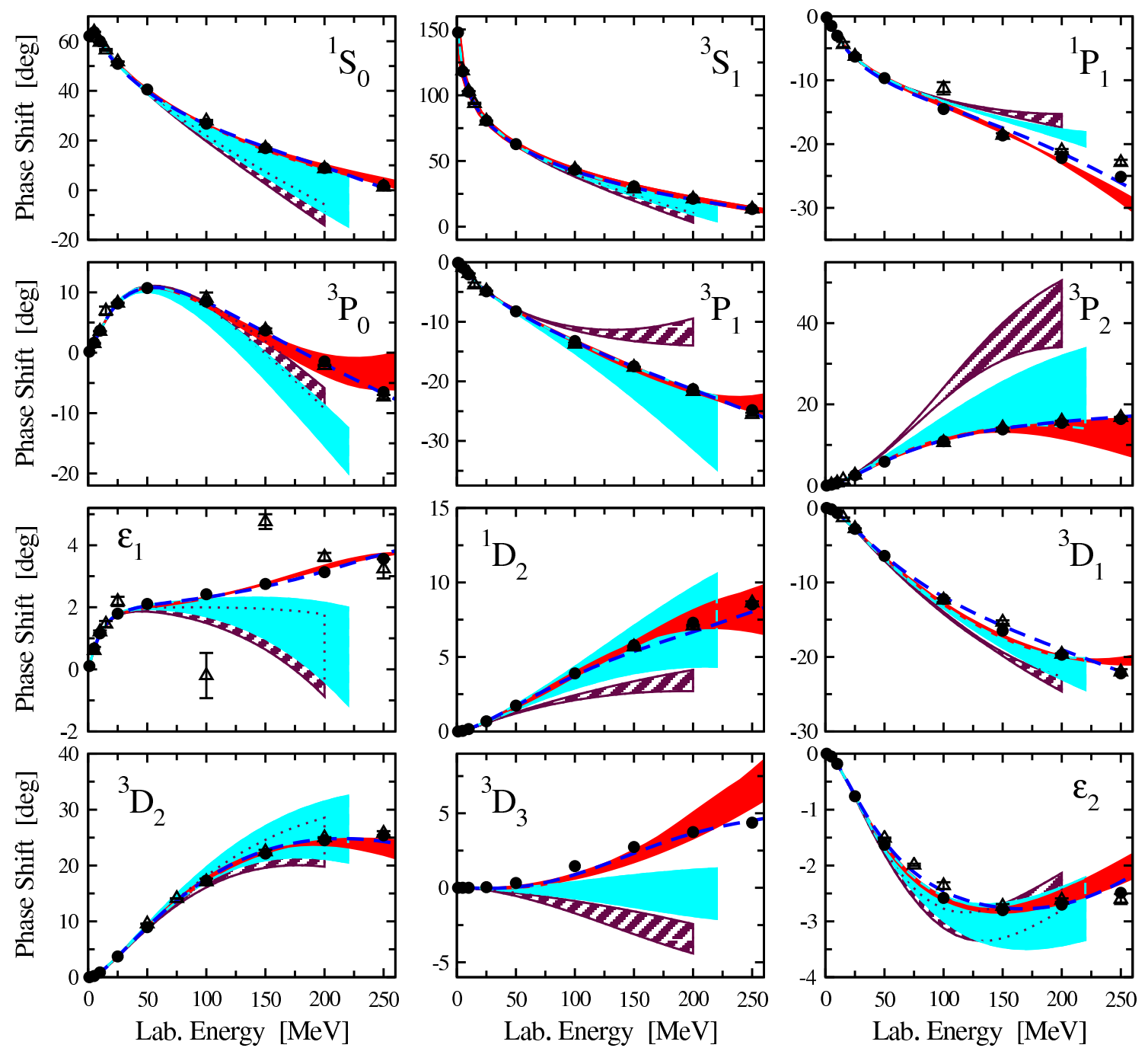

Figure 1: $\mathrm{S}-, \mathrm{P}-$ and $\mathrm{D}-$ waves $n p$ phase shifts. The dashed, light shaded and dark shaded bands show the NLO, $\mathrm{N}^{2} \mathrm{LO}$ and $\mathrm{N}^{3} \mathrm{LO}$ [18] results, respectively. The dashed line is the $\mathrm{N}^{3} \mathrm{LO}$ result of Ref. [19]. The filled circles (open triangles) depict the results from the Nijmegen multi-energy PWA [20, 21] (Virginia Tech single-energy PWA [22]).

Jakubowsky equations for more than four nucleons is a very difficult task. To solve the manybody problem we propose to put the chiral effective potential on the lattice and apply the powerful Monte-Carlo techniques which are already developed to high degree. In this framework, nucleons are represented as point-like Grassman-fields and pions as point-like instantaneous (in order to reproduce the chiral potential) pseudoscalar fields. Typically, our calculations are carried out using the lattice length $L \simeq 20 \mathrm{fm}$ and the lattice spacing $a \simeq 2 \mathrm{fm}$ which corresponds to the cutoff $\Lambda=\pi / a \simeq 300 \mathrm{MeV}$. The correlation function for $A$ nucleons in the Euclidean space is defined by

$$
Z_{A}(t)=\left\langle\Psi_{A}|\exp (-t H)| \Psi_{A}\right\rangle,
$$

where the states $\left|\Psi_{A}\right\rangle$ refer to the slater determinants for $A$ free nucleons, $H$ is the Hamiltonian of the system and $t$ the Euclidean time. The ground state energy of the $A$-nucleon system can be 

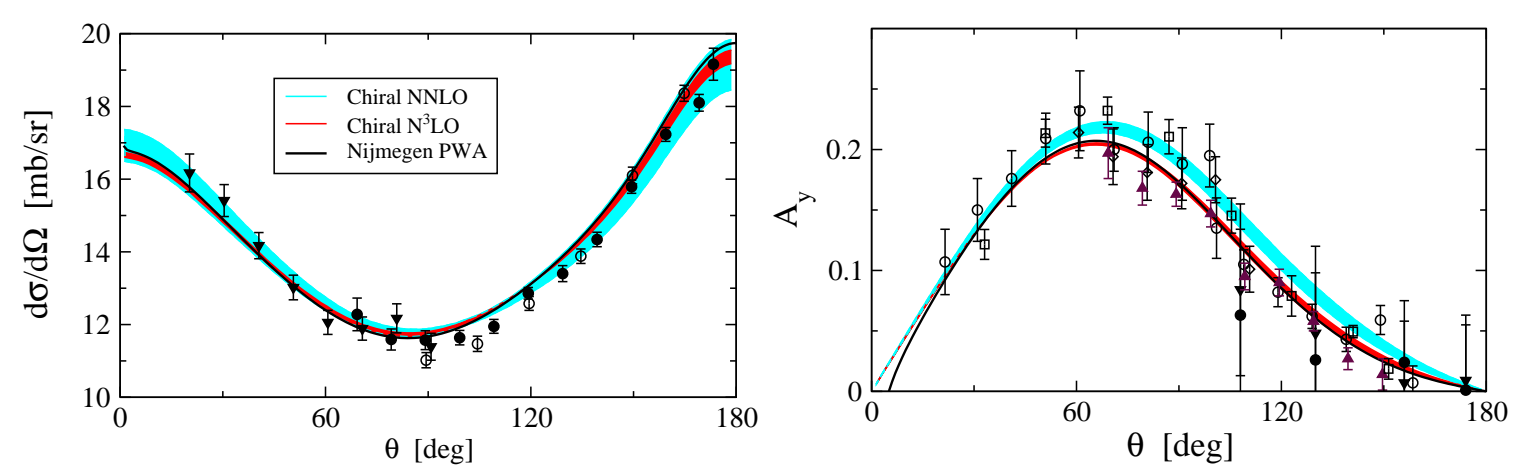

Figure 2: $n p$ differential cross section and vector analyzing power at $E_{\text {lab }}=50 \mathrm{MeV}$. The Nijmegen PWA result is taken from [21].

derived from the asymptotic behavior of the correlation function for large $t$.

$$
E_{A}^{0}=-\lim _{t \rightarrow \infty} \frac{d}{d t} \ln Z_{A}(t) .
$$

Expectation value of a normal ordered operator $\mathscr{O}$ can be derived in a similar way by

$$
\left\langle\Psi_{A}^{0}|\mathscr{O}| \Psi_{A}^{0}\right\rangle=\lim _{t \leftarrow \infty} \frac{Z_{A}^{\mathscr{O}}(t)}{Z_{A}(t)}, \quad Z_{A}^{\mathscr{O}}(t)=\left\langle\Psi_{A}|\exp (-t H / 2) \mathscr{O} \exp (-t H / 2)| \Psi_{A}\right\rangle
$$

where the states $\left|\Psi_{A}^{0}\right\rangle$ denote the ground states of $A$-nucleons system. It is convenient to describe NN contact interactions by standard bilinear nucleon density operators using the HubbardStratonovich transformation. Using the relation

$$
\exp \left(\rho^{2} / 2\right) \sim \int d s \exp \left(-s^{2} / 2-s \rho\right)
$$

one can express terms quadratic in the nucleon density operator $\rho$ as terms linear in $\rho$ in the presence of auxiliary background fields. In this representation, the full correlation function is related to the path integral over pions and auxiliary fields,

$$
Z_{A}(t) \sim \int \prod_{I=1,2,3} D \pi_{I} \prod_{i} D s_{i} \exp \left(-S_{\pi \pi}-S_{s s}\right)\left\langle\Psi_{A}\left|M^{\left(L_{t}-1\right)}\left(\pi_{I}, s_{i}\right) \cdots M^{(0)}\left(\pi_{I}, s_{i}\right)\right| \Psi_{A}\right\rangle .
$$

Here $S_{\pi \pi}$ and $S_{s s}$ are free actions for pions and auxiliary fields $s_{i}$ and $M^{(n)}$ is a transfer matrix defined as an $n$ 'th step in the temporal direction. Note since we only have linear nucleon density operators in the action the amplitude

$$
\left\langle\Psi_{A}\left|M^{\left(L_{t}-1\right)}\left(\pi_{I}, s_{i}\right) \cdots M^{(0)}\left(\pi_{I}, s_{i}\right)\right| \Psi_{A}\right\rangle
$$

is just a slater determinant of single nucleon matrix elements $\mathscr{M}_{i, j}$ with $i, j=1, \ldots, A$.

\section{Lattice EFT at leading order}

To be specific, we give here the leading order action starting with the free theory. The presentation here is somewhat sketchy. For an extensive discussion see [26]. The free auxiliary fields and 
pion actions are given by

$$
S_{s s}\left(s, s_{I}\right)=\frac{1}{2} \sum_{\vec{n}} s(\vec{n})^{2}+\frac{1}{2} \sum_{I=1}^{3} \sum_{\vec{n}} s_{I}(\vec{n})^{2}, \quad S_{\pi \pi}\left(\pi_{I}\right)=\frac{\alpha_{t}}{2} \sum_{I=1}^{3} \sum_{\vec{n}} \pi_{I}(\vec{n})\left(-\Delta+M_{\pi}^{2}\right) \pi_{I}(\vec{n}),
$$

where $M_{\pi}$ is the physical pion mass, $I$ denotes isospin indices and $\alpha_{t}=a_{t} / a$. For nucleons we use $O\left(a^{4}\right)$ improved free lattice Hamiltonian defined by

$$
H_{\text {free }}=\frac{1}{m} \sum_{k=0}^{3} \sum_{\vec{n}_{s}, \hat{l}_{s}, i, j} f_{k}\left[a_{i, j}^{\dagger}\left(\vec{n}_{s}\right)\left(a_{i, j}\left(\vec{n}_{s}+k \hat{l}_{s}\right)+a_{i, j}\left(\vec{n}_{s}-k \hat{l}_{s}\right)\right)\right],
$$

where $m$ is the nucleon mass, the operators $a_{i, j}^{\dagger}\left(\vec{n}_{s}\right)$ and $a_{i, j}\left(\vec{n}_{s}\right)$ are the nucleon creation and annihilation operators, $\vec{n}_{s}$ are spatial coordinates, $\hat{l}_{s}$ are spatial unit vectors, the indices $i$ and $j$ stay for spin and isospin indices, respectively, and the coefficients $f_{k}$ read:

$$
f_{0,1,2,3}=\frac{49}{2},-\frac{3}{4}, \frac{3}{40},-\frac{1}{180} .
$$

To define the interactions we introduce the nucleon-density operators with different spin/isospin polarizations

$$
\begin{gathered}
\rho^{a^{\dagger}, a}\left(\vec{n}_{s}\right)=\sum_{i, j} a_{i, j}^{\dagger}\left(\vec{n}_{s}\right) a_{i, j}\left(\vec{n}_{s}\right), \quad \rho_{I}^{a^{\dagger}, a}\left(\vec{n}_{s}\right)=\sum_{i, j, j^{\prime}} a_{i, j^{\prime}}^{\dagger}\left(\vec{n}_{s}\right)\left[\tau_{I}\right]_{j^{\prime}, j} a_{i, j}\left(\vec{n}_{s}\right), \\
\rho_{I, S}^{a^{\dagger}, a}\left(\vec{n}_{S}\right)=\sum_{i, i^{\prime}, j, j^{\prime}} a_{i^{\prime}, j^{\prime}}^{\dagger}\left(\vec{n}_{s}\right)\left[\sigma_{S}\right]_{i^{\prime}, i}\left[\tau_{I}\right]_{j^{\prime}, j} a_{i, j}\left(\vec{n}_{S}\right) .
\end{gathered}
$$

The transfer matrix for $n_{t}$-th step has, besides the free part, two important contributions:

$$
\begin{aligned}
M^{\left(n_{t}\right)} & =: \exp \left\{-H_{\text {free }} \alpha_{t}-\frac{g_{A} \alpha_{t}}{2 F_{\pi}} \sum_{S, I} \sum_{\vec{n}_{s}} \nabla_{S} \pi_{I}\left(\vec{n}_{s}, n_{t}\right) \rho_{S, I}^{a^{\dagger}, a}\left(\vec{n}_{s}\right)\right. \\
& \left.+\sqrt{-C \alpha_{t}} \sum_{\vec{n}_{s}}\left[s\left(\vec{n}_{s}, n_{t}\right) \rho^{a^{\dagger}, a}\left(\vec{n}_{s}\right)+i \sqrt{C_{I} \alpha_{t}} \sum_{I} s_{I}\left(\vec{n}_{s}, n_{t}\right) \rho_{I}^{a^{\dagger}, a}\left(\vec{n}_{s}\right)\right]\right\}:
\end{aligned}
$$

Here :: denotes normal ordering. The first long-range contribution includes the instantaneous pionnucleon interaction and describes the one-pion-exchange in the leading-order effective potential. The second short-range contribution corresponds to the $\mathrm{NN}$ contact interactions. The low-energy constants $C$ and $C_{I}$ fitted to Nijmegen PWA appear to have different signs:

$$
C<0, \quad C_{I}>0 .
$$

With these signs the pion-less theory appears to have no sign-oscillations if the number of protons and neutrons are equal and they stay pair-wise in isospin-singlet states. In this case the multiplication with $\tau_{2}$ of the single-nucleon matrix elements $\mathscr{M}$ from left and right is well defined and gives

$$
\tau_{2} \mathscr{M} \tau_{2}=\mathscr{M}^{*} .
$$

For this reason, the determinant of $\mathscr{M}$ appears to be real:

$$
\operatorname{det} \mathscr{M}^{*}=\operatorname{det} \mathscr{M} .
$$


Since $\tau_{2}$ is antisymmetric, the eigenvalues of $\mathscr{M}$ are doubly degenerate. This leads to a positive slater determinant $[8,27]$

$$
\operatorname{det} \mathscr{M} \geq 0 \text {. }
$$

The introduction of pions causes small sign-oscillations which, however, are not severe and appear to be suppressed.

To perform our simulations in a most efficient way we, in addition, exploit the approximate SU(4)-Wigner [28] symmetry in the NN system. The symmetry transformation is given by independent rotation of spin and isospin degrees of freedom.

$$
\delta N=\alpha_{\mu \nu} \sigma^{\mu} \tau^{v} N \quad \text { with } \quad \sigma^{\mu}=(1, \vec{\sigma}) \quad \text { and } \quad \tau^{\mu}=(1, \vec{\tau}) .
$$

One can show that in the limit where the NN S-wave scattering lengths approach infinity the twonucleon system becomes invariant under the SU(4)-transformation [29]. The SU(4)-breaking corrections come from the finite scattering length and higher order terms in the chiral expansion:

$$
\mathrm{SU}(4) \text { - breaking terms } \sim \frac{1}{a\left({ }^{3} S_{1}\right)}-\frac{1}{a\left({ }^{1} S_{0}\right)}, \frac{q}{\Lambda_{\chi}} .
$$

Since the NN scattering lengths

$$
a\left({ }^{1} S_{0}\right)=(-23.758 \pm 0.010) \mathrm{fm} \quad a\left({ }^{3} S_{1}\right)=(5.424 \pm 0.004) \mathrm{fm}
$$

are very large, the $\mathrm{SU}(4)$-breaking corrections appear to be small. This fact can be used to improve the performance of our lattice simulations. The $\mathrm{SU}(4)$ symmetric transfer matrix is given by

$$
M^{\left(n_{t}\right)}=: \exp \left[-H_{\text {free }} \alpha_{t}+\sqrt{-C \alpha_{t}} \sum_{\vec{n}_{s}} s\left(\vec{n}_{s}, n_{t}\right) \rho^{a^{\dagger}, a}\left(\vec{n}_{s}\right)\right]: .
$$

In this case there are no sign-oscillations for even number of nucleons [30] and we do have only one auxiliary field such that the simulations are much cheaper. Although there is no positivity theorem for odd numbers of nucleons, sign oscillations appear to be suppressed also in systems with odd number of nucleons because it is only one particle away from an even system with no sign-oscillation. Since the final result is closed to the one produced by SU(4)-symmetric simulation we divide our simulations in three parts. To simulate an expectation value of some observable we use SU(4)-symmetric transfer matrices in the first and the last $L_{t_{0}}$ steps in order to filter the lowenergy signal and after filtering start the simulation with realistic transfer matrices. A schematic overview of the transfer matrix calculation is shown in Fig. 3.

For our nuclear lattice simulations we use the hybrid Monte-Carlo (HMC) method [31]. We introduce the conjugate fields $p_{\pi_{I}}, p_{s}, p_{s_{I}}$ and use molecular dynamics trajectories to generate new configurations for the fields $p_{\pi_{I}}, p_{s}, p_{s_{I}}, \pi_{I}, s, s_{I}$ which keep the HMC Hamiltonian

$$
H_{\mathrm{HMC}}=\frac{1}{2} \sum_{\vec{n}}\left(\sum_{I}\left[p_{\pi_{I}}^{2}(\vec{n})+p_{s_{I}}^{2}(\vec{n})\right]+p_{s}^{2}(\vec{n})\right)+V\left(\pi_{I}, s, s_{I}\right),
$$

constant, where the HMC potential is defined by

$$
V\left(\pi_{I}, s, s_{I}\right)=S_{\pi \pi}+S_{s s}-\log |\operatorname{det} \mathscr{M}| .
$$

Upon completion of each molecular dynamics trajectory, we apply Metropolis accept or reject step for the new configuration according to the probability distribution $\exp \left(-H_{\mathrm{HMC}}\right)$. This process of molecular dynamics trajectory and Metropolis step is repeated many times. 


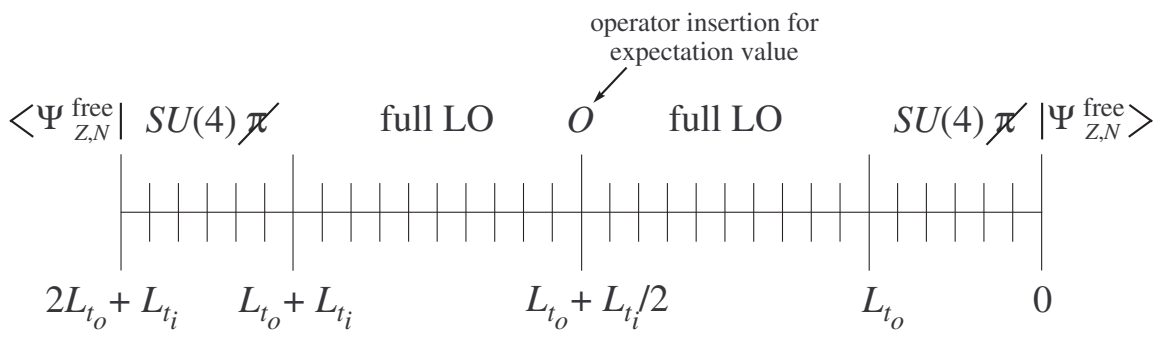

Figure 3: Overview of the various pieces of the transfer matrix calculation.

\begin{tabular}{||l||c|c|c|c||}
\hline \hline & $E_{3 \mathrm{H}}[\mathrm{MeV}]$ & $r_{3 \mathrm{H}}[\mathrm{fm}]$ & $E_{4 \mathrm{He}}[\mathrm{MeV}]$ & $r_{4 \mathrm{He}}[\mathrm{fm}]$ \\
\hline \hline \multirow{2}{*}{ Simulation } & $-8.9(2)$ & $2.27(7)$ & $-21.5(9)$ & $1.50(14)$ \\
Experiment & -8.482 & $1.755(9)$ & -28.296 & $1.673(1)$ \\
\hline \hline
\end{tabular}

Table 2: Experimental and nuclear lattice simulation results for binding energies and root-mean-square radius of triton and helium-4.

\section{Leading-order results}

With the presented method we performed nuclear lattice simulations on JUBL/JUGENE supercomputer at Forschungszentrum Jülich. Already at leading order we get promising results for binding energies, radii and density correlations for the deuteron, triton and helium-4 [26]. Numerical results on a $5^{3}$ lattice for triton and helium- 4 are shown in Table 2 . The triton binding energy agrees with experiment within 5\% and the triton root-mean-square radius is accurate to $30 \%$. The binding energy for helium- 4 is within $25 \%$ of the experimental value while the root-mean-square radius agrees within $10 \%$. Our results for the triton nucleon density correlations are shown in Fig. 4. We also studied the feasibility of simulations for light nuclei with up to eight nucleons and observed that for $A \leq 8$ the CPU time scales approximately linear with A.

\section{Next-to-leading-order results}

At NLO there appear 9 low energy constants (LECs) which we fitted to the Nijmegen NN scattering data. Elastic scattering phase-shifts on the lattice are related by Lüscher's [32, 33, 34] formula to the energy levels of two-body states in a finite large volume cubic box with periodic boundary conditions. While this method is very useful at low momenta, it is not so useful for determining phase shifts on the lattice at higher energies and higher orbital angular momenta. Furthermore, spin-orbit coupling and partial-wave mixing are difficult to measure accurately using Lüscher's method due to multiple-scattering artifacts produced by the periodic cubic boundary conditions. In [1] we proposed a more robust approach to measure phase shifts for two nonrelativistic point particles on the lattice using a spherical wall boundary. The basic idea is to impose 


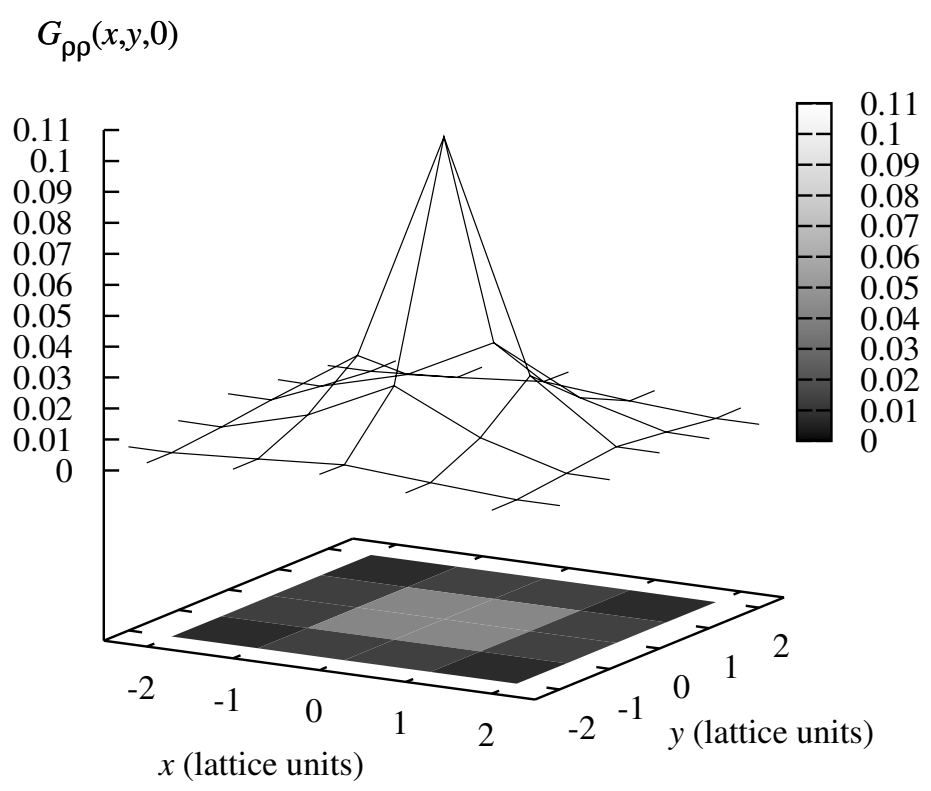

Figure 4: The nucleon density correlation for the triton in $x y$-plane.

a hard spherical wall boundary on the relative separation between the two interacting particles at some chosen radius. The reason for this spherical wall is to remove copies of the two-particle interactions due to the periodic boundaries on the lattice. This additional boundary condition allows for a direct extraction of the phase-shifts and mixing angles from the finite-volume spectrum. For more details see [1].

Using the spherical wall method we determined the values of 9 LECs by matching three Swave, four P-wave scattering data points, as well as deuteron binding energy and quadrupole moment. In Fig. 5 we show NN S-wave phase-shifts and mixing angles for two different actions, called $\mathrm{LO}_{1}$ and $\mathrm{LO}_{2}$. The action $\mathrm{LO}_{1}$ is the one presented in Eq. 5.6. In the action $\mathrm{LO}_{2}$ the contact interactions are smeared by a Gaussian. The two actions are identical at leading order and differ only by higher-order terms. The main motivation to introduction the Gaussian smearing was to cure a multi-particle clustering instability at coarse lattice spacing present in simulations with $\mathrm{LO}_{1}$ and to estimate a systematic error coming from higher-order corrections see [26] for extended discussion. As can be seen from Fig. 5, our lattice simulation results are in a good agreement with the partial wave results for momenta smaller than $80 \mathrm{MeV}$. Deviations between the two results for different actions appear merely at larger momenta and are consistent with the expected higher order effects.

\section{Dilute neutron matter}

As a first application at NLO we simulate dilute neutron matter in a periodic box [3]. We probe the density range from $2 \%$ to $8 \%$ of normal nuclear matter density. Neutron-rich matter at this density is likely to be present in the inner crust of neutron stars. The Pauli suppression of three-body forces in dilute neutron matter makes it a good testing ground for chiral EFT applied to 

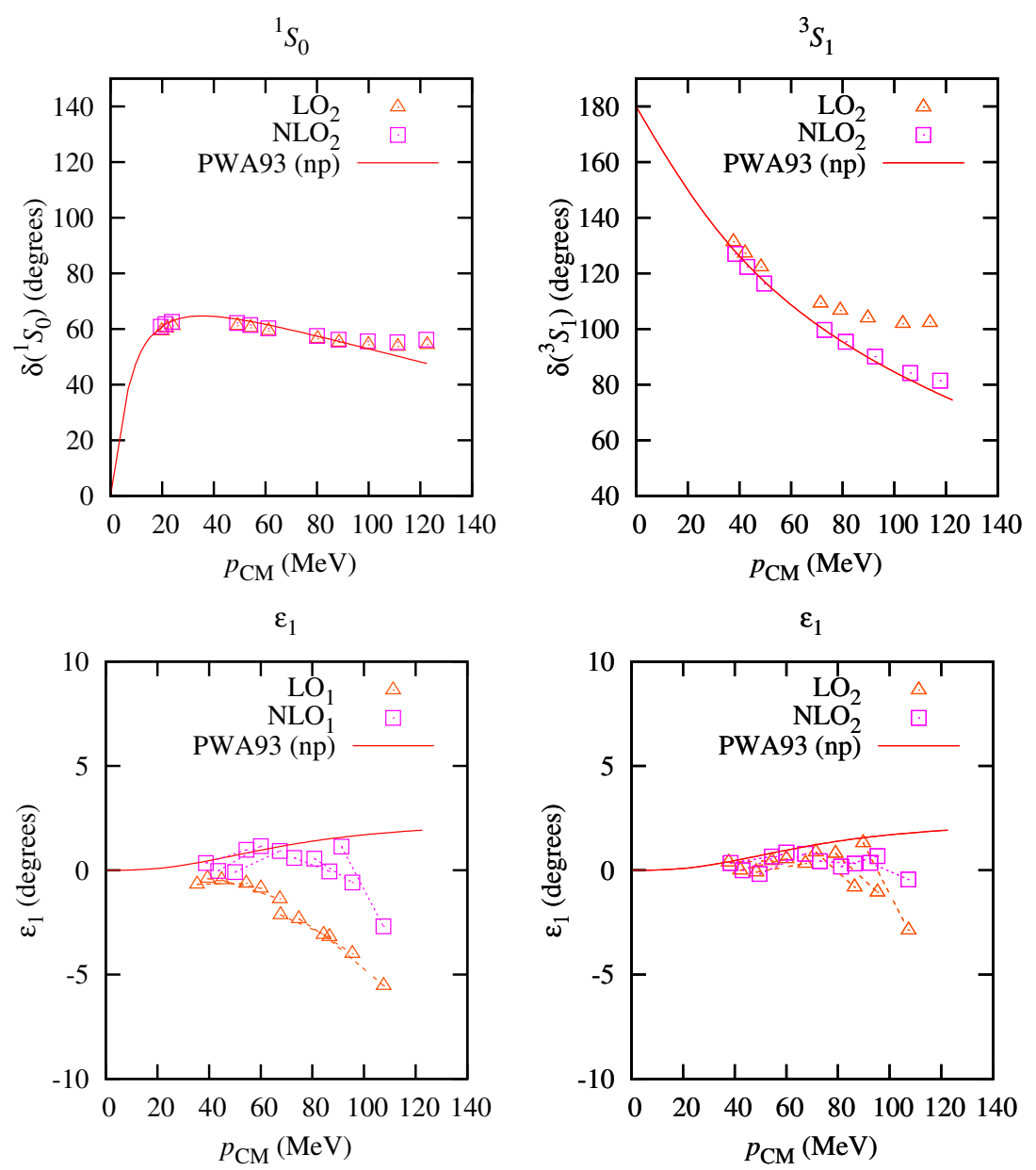

Figure 5: NN S-wave phase shifts and mixing angles versus center-of-mass momentum with actions $\mathrm{LO}_{1}$ and $\mathrm{LO}_{2}$.

many-nucleon systems. Neutron matter at $k_{F} \sim 80 \mathrm{MeV}$, where

$$
k_{F}=\frac{1}{L}\left(3 \pi^{2} N\right)^{1 / 3}
$$

is Fermi momentum, is close to the so-called idealized unitary limit. In this limit the S-wave scattering length is infinite and the range of the interaction is zero such that the scattering amplitude is as strong as possible. At lower densities corrections due to the finite scattering length become more important while at higher densities corrections due to effective range start to dominate. In the unitary limit the ground state has no dimensionful parameters other than the particle density and so the ground state energy of the system should obey a simple relation

$$
E_{0}=\xi E_{0}^{\text {free }}
$$

where $\xi$ is a dimensionless measurable constant. Due to its universal nature, the unitary limit can be studied in atomic systems. Ultracold ${ }^{6} \mathrm{Li}$ and ${ }^{40} \mathrm{~K}$ atoms e.g. can be tuned into the unitary limit by using a magnetic-field Feshbach resonance. Recently measured values for $\xi$ scatter considerably and have large error bars:

$$
\xi=0.51(4)[35], 0.46_{-05}^{+12}[36], 0.32_{-13}^{+10}[37] .
$$




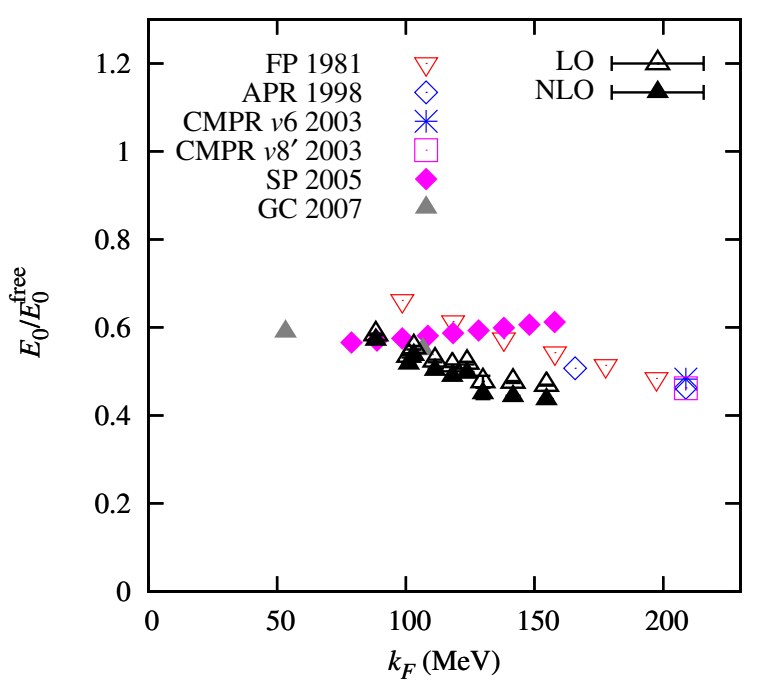

Figure 6: Results for $E_{0} / E_{0}^{\text {free }}$ versus Fermi momentum $k_{F}$. For comparison we show the results for FP 1981 [39], APR 1998 [40], CMPR $v 6$ and $v 8^{\prime} 2003$ [41], SP 2005 [42] and GC 2007 [43]

Earlier experiments tend to yield larger value for $\xi$ indicating the need of further experimental studies.

There have been numerous analytic calculations of $\xi$, see [38] for a recent review. The obtained values for $\xi$ vary roughly from 0.2 to 0.6 . To get a nuclear lattice EFT picture of the neutron matter in the unitary regime we simulate the ground state of 8,12 and 16 neutrons in a box of length $L=10,12$ and $14 \mathrm{fm}$ using Monte Carlo. In Fig. 6 we show ground energy ratio $E_{0} / E_{0}^{\text {free }}$ in dependence of Fermi momentum $k_{F}$. For comparison we also show earlier phenomenological calculations. Our predictions seem to be consistent with the earlier results. We find a good fit to the lattice data using

$$
E_{0} / E_{0}^{\mathrm{free}} \simeq \xi-\frac{\xi_{1}}{k_{F} a_{\mathrm{scatt}}}+0.16 k_{F} r_{\mathrm{eff}}-\left(0.51 \mathrm{fm}^{3}\right) k_{F}^{3} .
$$

The results from the fit are

$$
\xi \simeq 0.31 \text { and } \xi_{1} \simeq 0.81
$$

\section{9. $\mathbf{N}^{2}$ LO three-body forces}

At $\mathrm{N}^{2} \mathrm{LO}$ three-body forces start to show up which depend on two constants. We fit these LECs from neutron-deuteron scattering data in the spin- $1 / 2$ doublet channel and the triton binding energy. To describe the neutron-deuteron scattering we use standard Lüscher formula [32, 33, 34]. Finite volume spectrum was generated with Lanczos diagonalization method. In Fig. 7 we show the S-wave phase-shift in spin-1/2 doublet channel versus the square of the relative momentum and the triton binding energy versus the length of the lattice box. One observes a very natural convergence pattern in our simulations with increasing chiral order. Probing the triton binding energy we see 

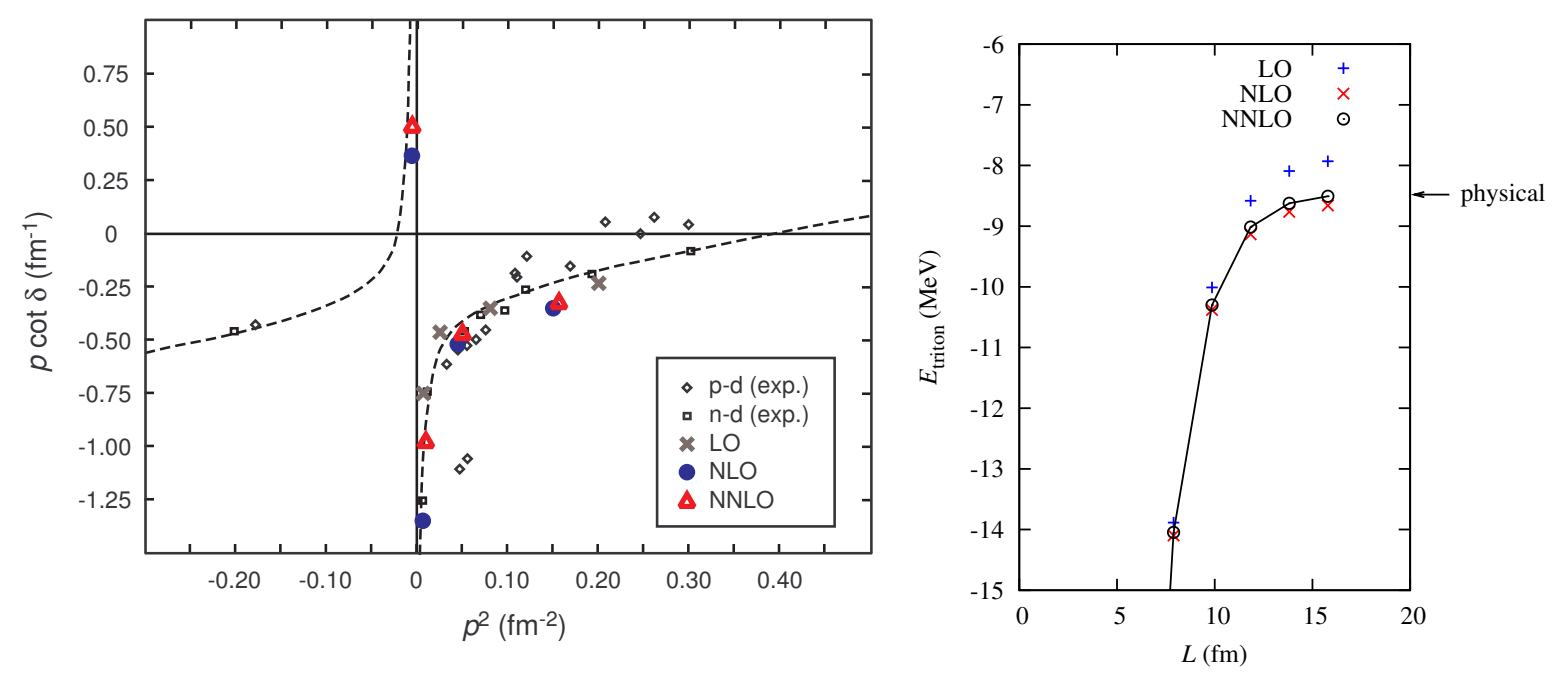

Figure 7: In the left panel: neutron-deuteron $S$-wave scattering phase-shifts in the spin-1/2 doublet channel versus the square of relative momentum. For completeness we show experimental data [44] for protondeuteron and neutron-deuteron scattering. In the right panel: triton binding energy versus the length of the lattice box.

from Fig. 7 that at the box length $\sim 15$ fm the volume dependence already becomes very small and the binding energy approaches its physical value. This is consistent with our expectation that the volume dependence in nuclear lattice EFT simulations should become weak for $L \sim 20 \mathrm{fm}$. In Fig. 8 we show the $\mathrm{S}$-wave phase-shifts in the spin-3/2 quartet channel versus the square of relative momentum. This channel was not taken into account in the fit procedure. Again we observe a very nice convergence with increasing chiral order. Our predictions are located between the protondeuteron and neutron-deuteron experimental data. Since the isospin-breaking was not taken into account in our simulations the results are very satisfactory.

As a first Monte-Carlo simulation of $\mathrm{N}^{2} \mathrm{LO}$ lattice EFT we studied the binding energy of ${ }^{4} \mathrm{He}$. The length of the box was chosen $L=16 \mathrm{fm}$. In Fig. 9 we show the resulting binding energy of the ${ }^{4} \mathrm{He}$ system

$$
\left\langle E_{4 \mathrm{He}}\right\rangle=\frac{\left\langle\Psi_{4}|\exp (-t H / 2) H \exp (-t H / 2)| \Psi_{4}\right\rangle}{\left\langle\Psi_{4}|\exp (-t H)| \Psi_{4}\right\rangle}
$$

versus Euclidean time $t$. Our Monte-Carlo simulations overpredict the physical binding energy with subtracted Coulomb-effects by $5 \%$. This is consistent with the expected theoretical accuracy of our simulations.

\section{Summary and outlook}

The results of our studies demonstrate that lattice EFT is a promising tool for a quantitative description of light nuclei. At leading order binding energies and radii of nuclei up to ${ }^{4} \mathrm{He}$ are reproduced with the accuracy $5 \ldots 30 \%$. At NLO, 9 LECs were fitted to the NN scattering phaseshift using the spherical wall method which is best suited to measure phase shifts and mixing 


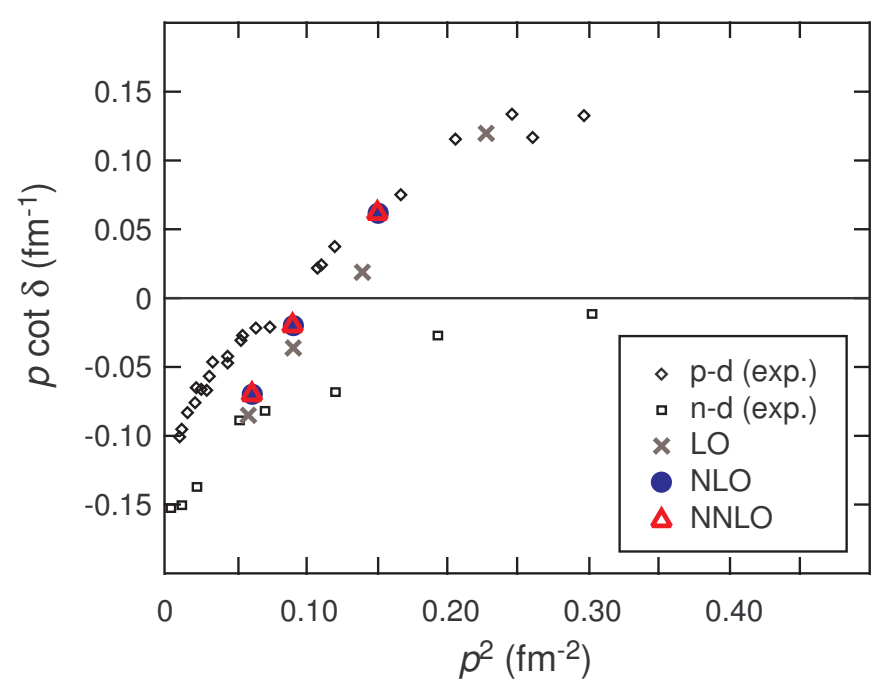

Figure 8: Neutron-deuteron scattering $S$-wave phase-shifts in the spin-3/2 quartet channel versus the square of relative momentum. The data for proton-deuteron and neutron-deuteron scattering are taken from [44].

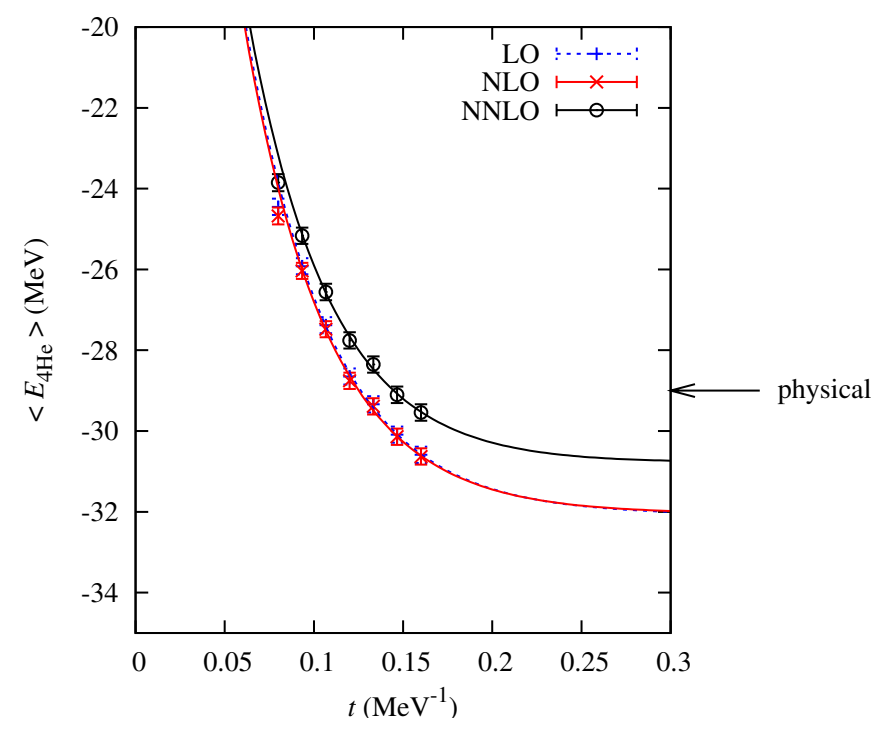

Figure 9: Binding energy expectation value of ${ }^{4} \mathrm{He}$ versus Euclidean time $t$. Plot produced by Monte-Carlo simulation with the box length $L=16 \mathrm{fm}$.

angles for nonrelativistic point particles on the lattice. With the NLO EFT action, we studied dilute neutron matter close to the unitary limit. We performed Monte-Carlo simulation with $N=8,12$ and 16 neutrons in a box of length $L=10,12$ and $14 \mathrm{fm}$. Our simulation results are consistent with earlier phenomenological determinations. We also presented the first analysis of $\mathrm{N}^{2} \mathrm{LO}$ lattice EFT. At this order, the two LECs entering the three-body force were fitted to neutron-deuteron scattering data and the triton binding energy. In our first $\mathrm{N}^{2} \mathrm{LO}$ Monte-Carlo simulation we calculated the binding energy of ${ }^{4} \mathrm{He}$. Our simulations overpredict the physical binding energy of ${ }^{4} \mathrm{He}$ by $\sim 5 \%$ 
which is within the expected accuracy of our lattice simulations.

In the future, we plan to perform $\mathrm{N}^{2} \mathrm{LO}$ Monte-Carlo simulations of light nuclei and probe neutron matter with larger number of neutrons in a box.

\section{Acknowledgments}

$\mathrm{H}$. K. is grateful to the organizers for invitation and making this exciting conference possible. Partial financial support from the Deutsche Forschungsgemeinschaft (SFB/TR 16), Helmholtz Association (contract number VH-NG-222 and VH-VI-231), and U.S. Department of Energy (DEFG02-03ER41260) are gratefully acknowledged. This research is part of the EU Integrated Infrastructure Initiative in Hadron Physics under contract number RII3-CT-2004-506078. The computational resources for this project were provided by the Jülich Supercomputing Centre at the Forschungszentrum Jülich.

\section{References}

[1] B. Borasoy, E. Epelbaum, H. Krebs, D. Lee and U.-G. Meißner, Eur. Phys. J. A 34, 185 (2007) [arXiv:0708.1780 [nucl-th]].

[2] B. Borasoy, E. Epelbaum, H. Krebs, D. Lee and U.-G. Meißner, Eur. Phys. J. A 35, 343 (2008) [arXiv:0712.2990 [nucl-th]].

[3] B. Borasoy, E. Epelbaum, H. Krebs, D. Lee and U.-G. Meißner, Eur. Phys. J. A 35, 357 (2008) [arXiv:0712.2993 [nucl-th]].

[4] D. Lee, arXiv:0804.3501 [nucl-th].

[5] R. Brockmann and J. Frank, Phys. Rev. Lett. 68 (1992) 1830.

[6] J. D. Walecka, Annals Phys. 83, 491 (1974).

[7] H. M. Muller, S. E. Koonin, R. Seki and U. van Kolck, Phys. Rev. C 61, 044320 (2000) [arXiv:nucl-th/9910038].

[8] J. W. N. Chen and D. B. Kaplan, Phys. Rev. Lett. 92, 257002 (2004) [arXiv:hep-lat/0308016].

[9] S. Chandrasekharan, M. Pepe, F. D. Steffen and U. J. Wiese, Nucl. Phys. Proc. Suppl. 129, 507 (2004) [arXiv:hep-lat/0309093].

[10] I. A. Shushpanov and A. V. Smilga, Phys. Rev. D 59, 054013 (1999) [arXiv:hep-ph/9807237].

[11] R. Lewis and P. P. Ouimet, Phys. Rev. D 64, 034005 (2001) [arXiv:hep-ph/0010043].

[12] B. Borasoy, R. Lewis and P. P. Ouimet, Nucl. Phys. Proc. Suppl. 128, 141 (2004) [arXiv:hep-lat/0310054].

[13] D. Lee, B. Borasoy and T. Schafer, Phys. Rev. C 70, 014007 (2004) [arXiv:nucl-th/0402072].

[14] J. Gasser and H. Leutwyler, Annals Phys. 158, 142 (1984).

[15] H. Leutwyler, Annals Phys. 235, 165 (1994) [arXiv:hep-ph/9311274].

[16] S. Weinberg, Nucl. Phys. B 363, 3 (1991).

[17] E. Epelbaum, Prog. Part. Nucl. Phys. 57, 654 (2006) [arXiv:nucl-th/0509032]. 
[18] E. Epelbaum, W. Glockle and U.-G. Meißner, Nucl. Phys. A 747, 362 (2005) [arXiv:nucl-th/0405048].

[19] D. R. Entem and R. Machleidt, Phys. Rev. C 68, 041001 (2003) [arXiv:nucl-th/0304018].

[20] V. G. J. Stoks, R. A. M. Kompl, M. C. M. Rentmeester and J. J. de Swart, Phys. Rev. C 48, 792 (1993).

[21] NN-Online program, M. C. M. Rentmeester et al., http://nn-online.org.

[22] SAID on-line program, R. A. Arndt et al., http://gwdac.phys.gwu.edu.

[23] C. Van Der Leun, C. Alderliesten, Nucl. Phys. A 380, 261 (1982).

[24] N. L. Rodning and L. D. Knutson, Phys. Rev. C 41, 898 (1990).

[25] T. E. O. Ericson and M. Rosa-Clot, Nucl. Phys. A 405, 497 (1983).

[26] B. Borasoy, E. Epelbaum, H. Krebs, D. Lee and U.-G. Meißner, Eur. Phys. J. A 31, 105 (2007) [arXiv:nucl-th/0611087].

[27] D. Lee, Phys. Rev. C 70, 064002 (2004) [arXiv:nucl-th/0407088].

[28] E. Wigner, Phys. Rev. 51, 947 (1937).

[29] T. Mehen, I. W. Stewart and M. B. Wise, Phys. Rev. Lett. 83, 931 (1999) [arXiv:hep-ph/9902370].

[30] J. W. Chen, D. Lee and T. Schafer, Phys. Rev. Lett. 93, 242302 (2004) [arXiv:nucl-th/0408043].

[31] S. Duane, A. D. Kennedy, B. J. Pendleton and D. Roweth, Phys. Lett. B 195 (1987) 216.

[32] M. Lüscher, Commun. Math. Phys. 104, 177 (1986).

[33] M. Lüscher, Commun. Math. Phys. 105, 153 (1986).

[34] M. Lüscher, Nucl. Phys. B 354, 531 (1991).

[35] J. Kinast, A. Turlapov, J. E. Thomas, Q. Chen, J. Stajic, and K. Levin, Science 307, 1296 (2005), cond-mat/0502087.

[36] J. T. Stewart, J. P. Gaebler, C. A. Regal, D. S. Jin, Phys. Rev. Lett. 97, 220406 (2006), [arXiv.org:cond-mat/0607776].

[37] M. Bartenstein, A. Altmeyer, S. Riedl, S. Jochim, C. Chin, J. Hecker Denschlag, R. Grimm, Rhys. Rev. Lett. 92, 10401 (2004).

[38] R. J. Furnstahl, G. Rupak and T. Schafer, arXiv:0801.0729 [nucl-th].

[39] B. Friedman and V. R. Pandharipande, Nucl. Phys. A 361 (1981) 502.

[40] A. Akmal, V. R. Pandharipande and D. G. Ravenhall, Phys. Rev. C 58, 1804 (1998) [arXiv:nucl-th/9804027].

[41] J. Carlson, J. . J. Morales, V. R. Pandharipande and D. G. Ravenhall, Phys. Rev. C 68, 025802 (2003) [arXiv:nucl-th/0302041].

[42] A. Schwenk and C. J. Pethick, Phys. Rev. Lett. 95, 160401 (2005) [arXiv:nucl-th/0506042].

[43] A. Gezerlis and J. Carlson, Phys. Rev. C 77, 032801 (2008) [arXiv:0711.3006 [nucl-th]].

[44] W. T. H. van Oers, J. D. Seagrave, Phys. Lett. B 24, 562 (1967). 